phys. stat. sol. (b) 229, No. 1, 433-437 (2002)

\title{
Confined Optical Vibrations in CdTe Quantum Dots and Clusters
}

\author{
A. G. Rolo ${ }^{1}$ ) (a), M. I. Vasilevskiy (a), N. P. Gaponik (b), A. L. Rogach (b), \\ and M. J. M. GoMes (a) \\ (a) Centro de Física, Universidade do Minho, Braga 4710-057, Portugal \\ (b) Institute of Physical Chemistry, University of Hamburg, Bundesstr. 45, \\ D-20146 Hamburg, Germany
}

(Received July 27, 2001; accepted September 30, 2001)

Subject classification: $63.22 .+\mathrm{m}$; 78.30.Fs; 78.67.-n; S8.13

\begin{abstract}
Absorption of far-infrared (FIR) radiation and Raman scattering in CdTe nano-crystals (spherical quantum dots (QDs) of several nanometers in size) and $\mathrm{Cd}_{54} \mathrm{Te}_{32}\left(\mathrm{SCH}_{2} \mathrm{CH}_{2} \mathrm{OH}\right)_{32}^{8-}$ clusters prepared by colloidal synthesis were studied. Raman spectra of both QDs and clusters are dominated by a peak situated slightly below the bulk CdTe LO phonon frequency. Experimental FIR transmittance spectra of films containing CdTe clusters show a broad band between the bulk TO and LO phonon frequencies, while there is only a single dip at the Fröhlich frequency observed for larger QDs. These observations are in agreement with the results of our numerical lattice dynamics calculations and previously published theoretical results based on a continuous model. It is suggested that the lattice dynamics of very small clusters is similar to that of large crystallites in the sense that there still exists the dispersion of optical vibration frequencies within the LO-TO band of bulk material.
\end{abstract}

Introduction II-VI semiconductor nanocrystals embedded in glass were the first quantum dots (QDs) to be studied [1,2]. Since that time, they have been produced by different techniques and extensively investigated. Wet chemistry methods have shown the ability to produce colloidal solutions of nearly spherical crystalline nanoparticles of various II-VI semiconductors with controllable size [3-6]. From such a solution, one can deposit films containing QDs embedded in a matrix or matrix-free (MF) films of near closely-packed QDs onto different substrates. In particular, it has become possible to produce extremelly small semiconductor clusters of some "magic" sizes, for example, $\mathrm{Cd}_{54} \mathrm{Te}_{32}\left(\mathrm{SCH}_{2} \mathrm{CH}_{2} \mathrm{OH}\right)_{32}^{8-}$ particles [7] (below referred to as $\mathrm{Cd}_{54} \mathrm{Te}$ clusters).

While the structure of QD electronic states is well understood based on the effective mass (EM) theory and (for smaller crystallites) numerical calculations, phonons confined in QDs (commonly referred to as "confined phonons") received much less attention until the last few years [8-12]. It has been shown theoretically $[10,11]$ that mechanical confinement indeed becomes important both for far-infrared (FIR) and Raman-active phonons in the limit of small QD size. Recently [12], we demonstrated the existence of spatially quantized dipolar modes for CdSe QDs of about $2 \mathrm{~nm}$ in diameter. Their frequencies were successfully calculated using the approach proposed in [10], which consists in solving the coupled continuous equation of motion and the Poisson equation. The validity of this semi-analytical model is, of course, a nice fact. However, it is unclear if it can be applied (if only qualitatively) to even smaller QDs and clusters. In other words, the question is whether bulk phonon dispersion curve parameters and TO-LO splitting have any relation to the vibrational properties of the clus-

\footnotetext{
1) Corresponding author; Fax: +351-253678981; e-mail: arolo@fisica.uminho.pt
} 
ters, or if it is just the anion-cation bond stretching frequency that determines the FIR and Raman-active vibrational modes in the clusters as suggested in [13].

In this communication, we present FIR and Raman spectroscopy results for CdTe QDs and $\mathrm{Cd}_{54} \mathrm{Te}$ clusters and compare them to those obtained by numerical lattice dynamics calculations. We will show that the predictions of the continuous model [10-12] are qualitatively valid even for extremelly small semiconductor clusters.

Experimental Both CdTe nano-crystals (NCs) and $\mathrm{Cd}_{54} \mathrm{Te}_{32}\left(\mathrm{SCH}_{2} \mathrm{CH}_{2} \mathrm{OH}\right)_{32}^{8-}$ clusters were produced by the aqueous synthesis described in [6]. Thioglycolic acid was used as a stabilizer for $\mathrm{CdTe} \mathrm{NCs}$, and 2-mercaptoethanol for $\mathrm{Cd}_{54} \mathrm{Te}$ clusters. Fractions of NCs with narrow $( \pm 10-15 \%)$ size distribution were separated using the size-selective precipitation technique [6] and re-dissolved in water again. The mean NC size ranged from 3 to $6 \mathrm{~nm}$ for different samples as determined from transmission electron microscopy studies and optical absorption measurements. The structure of $\mathrm{Cd}_{54} \mathrm{Te}$ clusters was proposed in [7] and their "size" was estimated as approximately $2.5 \mathrm{~nm}$. The CdTe particles (either NCs or clusters) were deposited on Si and silica substrates in the form of either MF films or composite layers of polyvinylalcohol (PVA) containing approximately $10 \%$ of CdTe (by volume). The volume fraction of semiconductor in the MF films was estimated to be 30-35\%, taking into account the organic capping layer.

We used FIR transmission and Raman spectroscopy to study the confined CdTe optical phonon modes. FIR transmittance measurements were performed at room temperature (RT) using a Bruker IFS66V FTIR spectrometer in the spectral range $50-700 \mathrm{~cm}^{-1}$ at normal incidence. The resolution was better than $2 \mathrm{~cm}^{-1}$. The transmittance spectra of two samples are shown in Figs. 3a, c.

RT Raman spectra (Figs. 3b, d) were measured with a Jobin-Yvon T64000 spectrometer in backscattering geometry using the 488 and $514.5 \mathrm{~nm} \mathrm{Ar}^{+}$laser lines (laser power on the sample was less than $0.3 \mathrm{~mW}$ ). The spot size and the spectral resolution were $\sim 1 \mu \mathrm{m}$ and $\sim 1 \mathrm{~cm}^{-1}$, respectively.

Calculated Results and Discussion The optical absorption spectra of CdTe NCs (Fig. 1, full curve) are typical for spherical II-VI QDs and can be modelled within the EM approximation (see, for example, [14]). This is not the case for the $\mathrm{Cd}_{54} \mathrm{Te}$ clusters. The

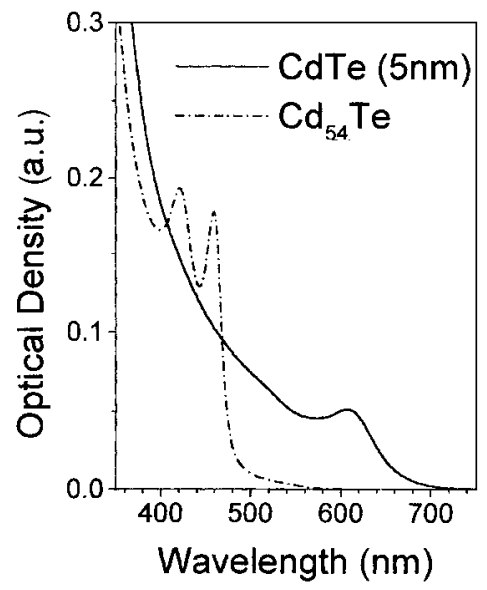
strong second absorption peak (Fig. 1, broken curve) cannot be explained by the EM theory, which does not reproduce correctly the structure of the electronic states in such small clusters. This is the reason why we tried to model the FIR and Raman spectra using numerical lattice dynamics calculations instead of applying the continuous model of $[10,11]$, which has been successful for CdSe and CdS QDs [12, 15].

Fig. 1. Optical absorption spectra of thin films containing $5 \mathrm{~nm}$ CdTe QDs (full curve) and $\mathrm{Cd}_{54} \mathrm{Te}$ clusters (broken curve) 
We studied the lattice dynamics problem in two dimensions using a model described in detail in [15]. It takes into account the short-range and Coulomb interatomic interactions. The interaction parameters were chosen to reproduce the bulk CdTe TO $\left(\omega_{\mathrm{TO}}\right)$ and LO $\left(\omega_{\mathrm{LO}}\right)$ phonon frequencies at the $\Gamma$ point when periodic boundary conditions were applied. Clusters imitating CdTe particles were generated on a square lattice from a perfect binary supercell replacing some $\mathrm{Cd}$ and $\mathrm{Te}$ atoms by very heavy chargeless particles outside the cluster boundary, thus imposing nearly rigid mechanical boundary conditions. An example of such a cluster is shown in the inset of Fig. 2. By diagonalizing the supercell dynamical matrix, we obtained all the vibrational modes of the system. Given these, the cluster polarizability (normalized by its "volume") was calculated according to the expression

$$
\alpha=-\frac{\mu}{2 \pi}\left(\omega_{\mathrm{LO}}^{2}-\omega_{\mathrm{TO}}^{2}\right) \sum_{v}\left\{\left[\sum_{l, k} \frac{(-1)^{k} B_{v}(l, k)}{\sqrt{M(l, k)}}\right]^{2} \frac{1}{\omega^{2}-\omega_{v}^{2}+i \delta^{2}}\right\},
$$

where $\mu$ is the reduced atomic mass, $v$ runs over eigenmodes $\left(\omega_{v}^{2}\right), l$ and $k$ enumerate unit cells and two sublattices, respectively, $M(l, k)$ are atomic masses, $B_{v}(l, k)$ is the $v$-th eigenvector and $\delta$ a (small) damping parameter. The expression used to calculate the Raman intensity has been given in [15].

Calculated extinction (imaginary part of the polarizability) and Raman spectra are shown in Fig. 2 for several cluster sizes. The Raman peak, which is situated practically at $\omega_{\mathrm{LO}}$ for the largest crystallites, shifts downwards and broadens (if normalized in intensity) when the cluster size decreases. One can also see that, in all cases, there are several confined modes contributing to the scattering. (We would like to note that the apparently two-mode structure of the Raman spectra for smaller cluster sizes in Fig. $2 \mathrm{~b}$ is specific to the parameters used in this calculation. In general, there are several modes contributing to the Raman lineshape.) This is also true for the extinction spectra.
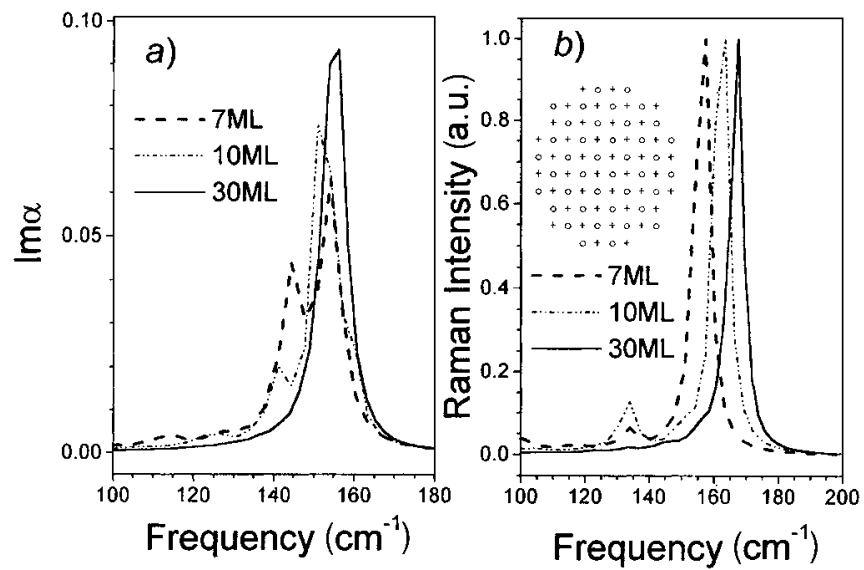

Fig. 2. a) Imaginary part of the polarizability defined by Eq. (1) and b) Raman intensity calculated numerically for round CdTe clusters of different diameter (given as the number of atomic monolayers, ML). The Raman intensity is normalized by its maximum value for each spectrum. The inset shows an example of the cluster where crosses and circles represent $\mathrm{Cd}$ and $\mathrm{Te}$ atoms, respectively 
However, for large (30 ML) crystallites, the contributions of all confined modes result in a single peak located at $\omega_{0}^{2}=\left(\omega_{\mathrm{LO}}^{2}+\omega_{\mathrm{TO}}^{2}\right) / 2$, which plays the role of the Fröhlich frequency in the present model. This agrees with the conclusion made in [12] on the basis of the continuous model. For very small clusters, several vibrational modes distributed throughout the CdTe reststrahl band produce comparable FIR absorption.

Let us turn to the experimental spectra of Fig. 3. Both for $5 \mathrm{~nm} \mathrm{NCs}$ and $\mathrm{Cd}_{54} \mathrm{Te}$ clusters, the Raman spectra show a broadened and slightly downshifted peak near the bulk LO phonon frequency, the shift and broadening being somewhat larger for the latter. Our calculations predict much larger shift for small clusters, but this is a shortcoming of the simplified lattice dynamics model. The transmittance spectrum of the film containing $5 \mathrm{~nm}$ NCs represents a single dip at the Fröhlich frequency, as expected from both the theory and numerical calculations. For the $\mathrm{Cd}_{54} \mathrm{Te}$ clusters, there is a broad absorption band seen between $\omega_{\mathrm{TO}}$ and $\omega_{\mathrm{LO}}$. Unfortunately, the presence of the stabilizer layer and/or matrix possessing some FIR activity does not permit to resolve the structure of this band, but still, qualitatively, this is what one should expect to observe according to our calculated results.

In conclusion, we have shown that Raman spectra of chemically prepared CdTe crystallites are dominated by a peak slightly below the bulk LO phonon frequency even in
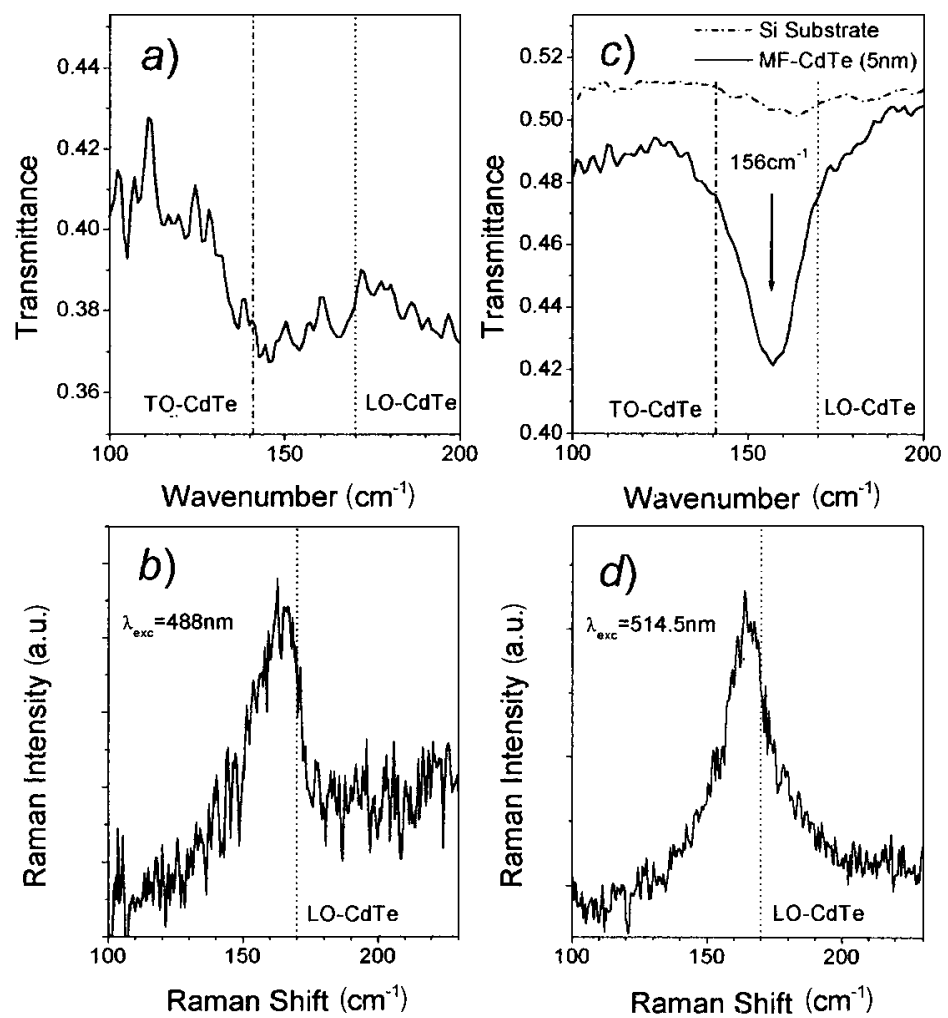

Fig. 3. a), c) Experimental FIR transmittance and b), d) Raman spectra of films containing a), b) $\mathrm{Cd}_{54} \mathrm{Te}$ clusters and c), d) $5 \mathrm{~nm}$ CdTe QDs 
the limit of very small clusters. The FIR absorption spectra of thin films containing such clusters show a broad band between the bulk TO and LO phonon frequencies. This implies that the lattice dynamics of such small clusters are similar to that of crystals in the sense that there still exists the dispersion of optical phonon frequencies and LO-TO splitting resulting from the Coulomb interaction between the atoms of two sublattices. When the NC size increases, the FIR absorption spectra produced by many vibrational modes converge to the one predicted by the electrostatic theory for spherical QDs [16], i.e., to a single absorption peak at the Fröhlich frequency.

Acknowledgements This work was supported by FCT-PRAXIS (Portugal). The authors wish to thank L.G. Vieira and J.L. Ribeiro for help in performing FIR transmittance measurements.

\section{References}

[1] A.I. Ekimov and A.A. Onuschenko, Sov. Phys. - Semicond. 16, 1215 (1982).

[2] T. Arai, H. Fijumira, I. Umezu, T. Ogawa, and A. FujiI, Jpn. J. Appl. Phys. 28, 484 (1989).

[3] A.P. Alivisatos, J. Chem. Phys. 100, 13226 (1996).

[4] C.B. Murray, D.J. Norris, and M.G. Bawendi, J. Am. Chem. Soc. 115, 8706 (1993).

[5] M.V. Artemyev, A.I. Bibik, L.I. Gurinovich, S.V. Gaponenko, and U. WogGon, Phys. Rev. B 60, 1504 (1999).

[6] A.L. Rogach, L. Katsikas, A. Kornowski, D. Su, A. Eychmüller, and H. Weller, Ber. Bunsenges. Phys. Chem. 100, 1772 (1996).

[7] J. Rockenberger, L. Tröger, A.L. Rogach, M. Tischer, M. Grundmann, A. Eychmüller, and H. Weller, J. Chem. Phys. 108, 7807 (1998).

[8] S. Nomura and T. Kobayashi, Phys. Rev. B 45, 1305 (1992).

[9] T.D. Krauss, F.W. Wise, and D.B. Tanner, Phys. Rev. Lett. 76, 1376 (1996).

[10] E. Roca, C. Trallero-Giner, and M. Cardona, Phys. Rev. B 49, 13704 (1994).

[11] M.P. Chamberlain, C. Trallero-Giner, and M. Cardona, Phys. Rev. B 51, 1680 (1995).

[12] M.I. Vasilevskiy, A.G. Rolo, M.V. Artemyev, S.A. Filonovich, M.J.M. Gomes, and Yu.P. RakoVICH, phys. stat. sol. (b) 224, 599 (2001).

[13] T. Lover, G.A. Bowmaker, J.M. SEakins, and R.P. Cooney, Chem. Mater. 9, 967 (1997).

[14] M.I. Vasilevskiy, A.M.P. De Paula, E.I. Akinkina, and E.V. Anda, Semiconductors 32, 1229 (1998).

[15] M.I. Vasilevskiy, A.G. Rolo, M.J.M. Gomes, O.V. Vikhrova, and C. Ricolleau, J. Phys.: Condens. Matter 13, 3491 (2001).

[16] R. Ruppin and R. Englman, Rep. Prog. Phys. 33, 149 (1970). 
